\title{
Ultrafast Interaction of the Angular Momentum of Photons with Spins in the Metallic Amorphous Alloy GdFeCo
}

\author{
C. D. Stanciu, ${ }^{1, *}$ F. Hansteen, ${ }^{1}$ A. V. Kimel, ${ }^{1}$ A. Tsukamoto, ${ }^{2}$ A. Itoh, ${ }^{2}$ A. Kirilyuk, ${ }^{1}$ and Th. Rasing ${ }^{1}$ \\ ${ }^{1}$ Institute for Molecules and Materials, Radboud University Nijmegen, Toernooiveld 1, 6525 ED Nijmegen, The Netherlands \\ ${ }^{2}$ College of Science and Technology, Nihon University, 7-24-1 Funabashi, Chiba, Japan \\ (Received 10 February 2006; revised manuscript received 7 March 2007; published 14 May 2007)
}

\begin{abstract}
Ultrashort laser pulses have been used to study the effect of circularly polarized light on spins in the ferrimagnetic metal GdFeCo. By turning the sample into a multidomain state and thereby suppressing the observation of the heating effect of light, we have been able to demonstrate an ultrafast nonthermal excitation of spin waves with a phase that depends on the angular momentum of the photons. These results demonstrate the possibility of ultrafast coherent control of the magnetization in this metallic system.
\end{abstract}

DOI: 10.1103/PhysRevLett.98.207401

PACS numbers: 78.47.+p, 75.50.Gg, 75.50.Ss, 76.50.+g

The strongly nonequilibrium conditions developed in magnetic materials following excitation by intense femtosecond laser pulses represent a subject that has attracted continuous growing interest over the last two decades [120]. However, due to this strong nonequilibrium, the conventional description of magnetic phenomena in terms of thermodynamics is no longer valid. As a result, the ultrafast channels for transferring energy and angular momentum between photons, electrons, spins, and phonons remain elusive and a subject of debate [1].

In metallic magnets, the absorbed ultrashort laser pulses excite collective modes (plasmons) and electron-hole pairs (quasiparticles) within tens of femtoseconds, which acquire a large kinetic energy far above the Fermi level $[2,3]$. The quasiparticles then thermalize to a hot FermiDirac distribution due to electron-electron, hole-hole, and electron-hole scattering processes, on a time scale of a few hundred fs [2]. During this thermalization time an ultrafast demagnetization may occur [4]. This process is followed by a quasistatic equilibrium at which the spins follow the electronic temperature [5]. Subsequently, the electronphonon interaction leads to an increase of the lattice temperature while the electrons cool down $(t<2 \mathrm{ps})$ [6]. Throughout this Letter, the above described processes will be referred to as heat-driven effects. Surprisingly, it has been reported that laser excitation of a magnetic metallic film may lead to a demagnetization of the material within $50 \mathrm{fs}$, thus much faster than the typical spin-spin and spin-phonon characteristic times [7]. Furthermore, it has recently been demonstrated that light can excite the metallic spin system also before electron thermalization, via excitation of coherent optical phonons [8]. Such intriguing observations raise fundamental questions about the mechanisms responsible for the ultrafast optical excitation of spins in metals.

In this context it has been suggested that in a metallic magnet, photons may also excite spins nonthermally via an ultrafast interaction between the angular momentum of the photons and the spin system [9]. However, it has been noted that the number of photons involved in real experiments is too low to support such ultrafast action of the angular momentum on the spin system in metals, based on the transfer of photon angular momentum via absorption [10]. Indeed, several attempts to detect an ultrafast effect of the light helicity in ferromagnetic metals such as $\mathrm{Ni}$ and other transition metals have remained unsuccessful [1113] and heat-driven effects of light were found to dominate in all these experiments.

In this Letter we demonstrate that via careful tuning of the conditions in all-optical pump-probe experiments, in particular, turning the sample into a multidomain state, it is possible to minimize the observation of the usually dominating heat-driven effects of light on the magnetization in the metallic ferrimagnet GdFeCo. Consequently, an ultrafast nonthermal interaction mechanism between photons and spins is revealed where the phase of the excited spin wave is given by the photon angular momentum. The ultrashort laser pulse acts on the ensemble of strongly correlated spins as a magnetic field pulse [14,15]. It is suggested that this optomagnetic mechanism takes place not by absorption of the angular momentum of the photons but via a Raman-like coherent optical scattering process. Therefore, it does not require annihilation of photons. It follows that one single photon can effectively interact with more than one spin, in this way yielding an efficient interaction between photons and spins.

The material chosen for this study was the ferrimagnetic alloy $\mathrm{GdFeCo}$, grown by magnetron sputtering in the following multilayer structure: glass/AlTi(10 nm)/ $\mathrm{SiN}(5 \mathrm{~nm}) / \mathrm{Gd}_{22} \mathrm{Fe}_{74.6} \mathrm{Co}_{3.4}(20 \mathrm{~nm}) / \mathrm{SiN}(60 \mathrm{~nm})$. The AlTi layer serves as a heat sink. The GdFeCo layer is an amorphous alloy characterized by a strong perpendicular anisotropy and a Curie temperature of about $500 \mathrm{~K}$.

Laser induced spin dynamics was investigated using a pump-probe technique. We have applied an external and nearly in-plane magnetic field $\mathbf{H}_{e}$ and excited the ferrimagnetic film by circularly polarized 100 fs laser pulses of $0.87 \mathrm{eV}$ photon energy from an amplified Ti:sapphire laser 


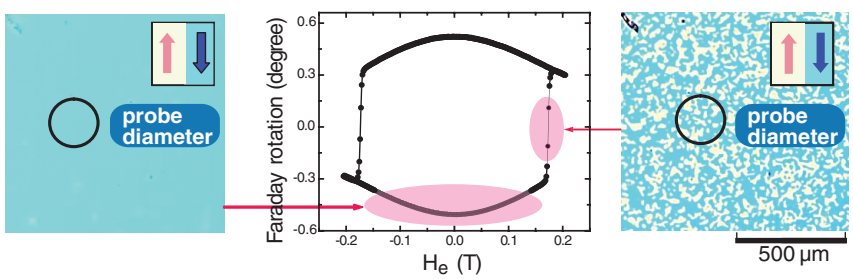

FIG. 1 (color online). The initial magnetic state of the GdFeCo sample is a single domain state (left image). Certain values of an external field applied at an angle from the normal to the sample turn the sample into a multidomain state (right image) with magnetic domains much smaller than the probe spot diameter. The images where captured by means of a polarization microscope.

with a repetition rate of $1 \mathrm{kHz}$. A less intense linearly polarized probe beam of the same energy was used to detect the changes in the magnetic system induced by the pump via measurements of the magneto-optical Faraday effect. The pump and probe beams had an intensity ratio of about 100 . At nearly normal incidence, the beams were focused on the sample to a spot of $200 \mu \mathrm{m}$ diameter for the pump and somewhat smaller for the probe beam. The present geometry is sensitive to the out-of-plane component of the magnetization, $\mathbf{M}_{z}$. The measurements were performed at room temperature.

The laser fluence used in our experiments to excite coherent precession of the magnetization in $\mathrm{GdFeCo}$ [16] was relatively high (about $2 \mathrm{~mJ} / \mathrm{cm}^{2}$ ). Hence, heat-driven effects of light on the magnetic order in the metallic ferrimagnet $\mathrm{GdFeCo}$ could be very strong. In order to suppress the observation of such heating effects and thus to allow observation of nonthermal effects, we used the concept of turning the sample into a multidomain state.

As shown in Fig. 1, the initial magnetic state of our sample is a single domain state. The application of an outof-plane field of opposite orientation with respect to the magnetization would reverse the magnetization via a few large $(>100 \mu \mathrm{m})$ domains, while the simultaneous presence of an in-plane external magnetic field results in the nucleation of a large numbers of small domains [21], with sizes much smaller than the probe area (see Fig. 1). Because of the coercivity, these magnetic domains are sufficiently stable in time and thus suitable for the stroboscopic pump-probe experiments. The balance between magnetocrystalline anisotropy field $\mathbf{H}_{a}$, shape anisotropy field $\mathbf{H}_{s}$, and external field $\mathbf{H}_{e}$ creates an initial state for these experiments such that the magnetization in each of the domains will be tilted [see Fig. 2(a)]. Laser-heating excitation of such a sample induces locally a change of the magnetocrystalline and shape anisotropy due to heating. This leads to a change of the equilibrium orientation for the magnetization and triggers a precession of the magnetic moments of the domains. These precessions proceed in such a way that the $z$ components of the magnetization in oppositely oriented domains (like $\mathbf{M}_{1}$ and $\mathbf{M}_{2}$ in a) Initial multidomain magnetic state

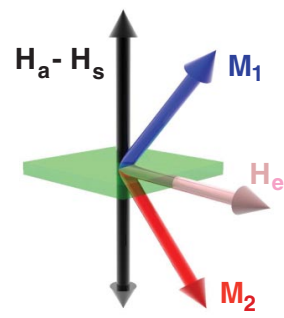

$\underline{\Delta t<0}$

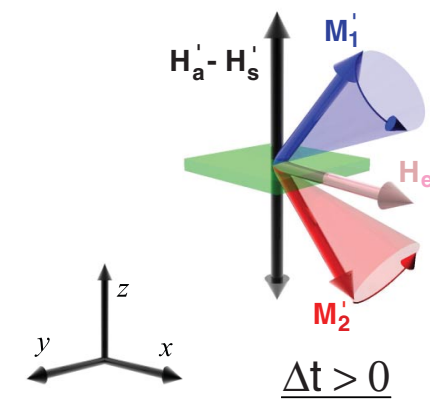

b) Heat-driven excitation

FIG. 2 (color online). (a) At $\Delta t<0$, the magnetizations $\mathbf{M}_{1}$ and $\mathbf{M}_{2}$ of oppositely magnetized domains are tilted due to the balance between magnetocrystalline anisotropy field $\mathbf{H}_{a}$, shape anisotropy field $\mathbf{H}_{s}$, and external field $\mathbf{H}_{e}$. (b) After photoexcitation, $\Delta t>0$, heating induces out of phase oscillations of the $z$ components of $\mathbf{M}_{1}$ and $\mathbf{M}_{2}$.

[Fig. 2(b)]) always oscillate out of phase. Since the probe beam is averaging over a large number of such oppositely oriented domains, the heat-driven effect of light on the magnetization is effectively averaged out in the Faraday signal by achieving $\sum \delta \mathbf{M}_{1}^{i}+\sum \delta \mathbf{M}_{2}^{j} \approx 0$, where the sums are taken over all the domains within the probed area. Experimentally, the optimum cancellation is achieved by adjusting the external magnetic field $\mathbf{H}_{e}$.

The results of these time-resolved measurements of the magneto-optical Faraday effect for right- $\sigma^{+}$and lefthanded $\sigma^{-}$circularly polarized pump pulses at different $\mathbf{H}_{e}$ are shown in Fig. 3. The variation of the magnetooptical signal $\Delta \theta_{F}$ representing the oscillatory behavior of $\mathbf{M}_{z}$ was plotted relative to the total Faraday rotation of the sample $\theta_{F}$. On the scale of 500 ps one can clearly distinguish two different processes. At zero time delay, instantaneous changes of the Faraday rotation are observed that result partly from ultrafast demagnetization and partly from changes of the magnetocrystalline anisotropy [6]. These instantaneous changes of the Faraday rotation are followed by oscillations with a frequency of about $7 \mathrm{GHz}$, which can clearly be ascribed to precession of the magnetization [16]. The strong damping observed can be understood as a result of the averaging over the many domains. Figure 3 shows that the difference between the magnetooptical Faraday signal for right- and left-handed excitation strongly depends on the external applied field $\mathbf{H}_{e}$. At $\mathbf{H}_{e}=$ $0.172 \mathrm{~T}$ the $\sigma^{+}$and $\sigma^{-}$pump pulses excite precession of opposite phase. Because the phase of the spin precession is given by the angular momentum of the exciting photons, this observation provides a first indication of an optomagnetic effect in the metallic ferrimagnet GdFeCo. An alternative interpretation of this observation including independent sublattice magnetization dynamics can not be valid. This is because far from the ferrimagnetic compen- 


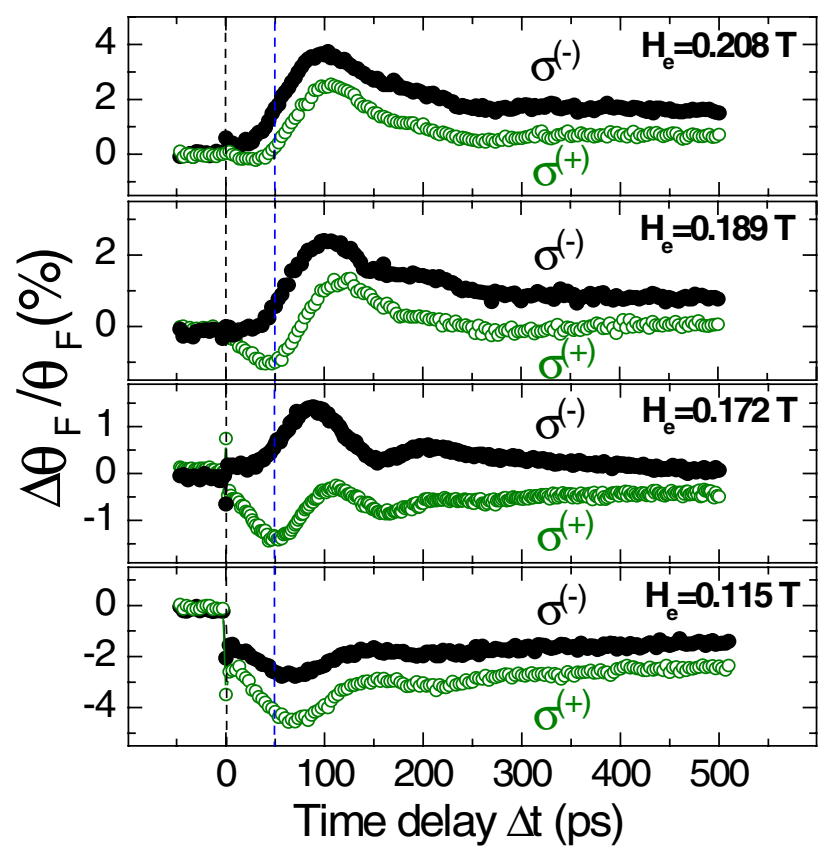

FIG. 3 (color online). Precession of the magnetization excited by circularly polarized pump pulses in $\mathrm{GdFeCo}$, probed via the magneto-optical Faraday effect. For certain values of the external field $\mathbf{H}_{e}$, the two helicities $\sigma^{+}$and $\sigma^{-}$give rise to precession with different phase. The lines are guides to the eye.

sation point (as is the case for the present experiments), $\mathrm{GdFeCo}$ behaves as a simple ferromagnet (see $[16,17]$ ).

In order to better separate this optomagnetic effect from the heat-driven one, we have calculated the sum and the difference of the magneto-optical signals resulting from excitation with $\sigma^{+}$and $\sigma^{-}$pump pulses. While the sum of the magneto-optical signals shows the helicity independent heat-driven effect of light on the magnetization $\Delta M_{\text {heat }} \sim$ $\Delta \theta_{F}\left(\sigma^{-}\right)+\Delta \theta_{F}\left(\sigma^{+}\right)$, the difference corresponds to the optomagnetic effect $\Delta M_{\text {opto }} \sim \Delta \theta_{F}\left(\sigma^{-}\right)-\Delta \theta_{F}\left(\sigma^{+}\right)$[11]. The results of the thus analyzed experimental data are plotted in Fig. 4. In the optomagnetic signal [Fig. 4(a)] one can identify an instantaneous change in $\Delta \theta_{F}$ after excitation. Although the origin of this instantaneous change is the demagnetization, which is a heat-driven effect, its observation here is a result of the optomagnetic effect. More specifically, the $\sigma^{+}$and $\sigma^{-}$pump pulses act on the magnetization in opposite directions resulting in different projections of the demagnetization process on the $\mathbf{k}$ vector of the probe beam. This will indeed lead to the observation of a step in $\Delta M_{\text {opto }}$ as observed in Fig. 4(a). As the strength of $\mathbf{H}_{e}$ increases, the magnetization is pulled towards the plane of the sample and this difference is gradually reduced. It can also be seen that the sign of the optomagnetic effect does not depend on the value of $\mathbf{H}_{e}$ while the heat-driven one [Fig. 4(b)] is characterized by a strong field dependence and changes sign at $\mathbf{H}_{e} \approx$ $0.172 \mathrm{~T}$. Since the amplitude of the heat-driven effect is strongly reduced at $\mathbf{H}_{e}=0.172 \mathrm{~T}$, it is clear that around

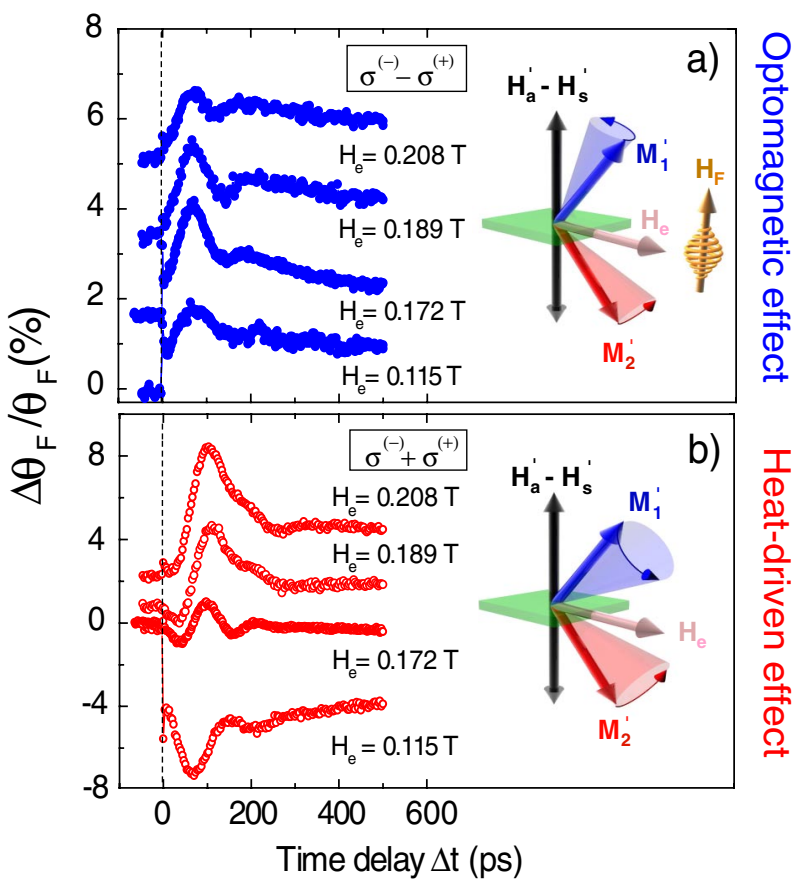

FIG. 4 (color online). The difference (a) and the sum (b) of the experimental data resulting from excitations by $\sigma^{+}$and $\sigma^{-}$ pump pulses, representing the optomagnetic and the heat-driven excitation, respectively. The curves are vertically displaced for clarity. The insets of the figure are schematic representations of the optomagnetic and the heat-driven excitation mechanisms.

this point the averaging out of the heating effect in the Faraday signal is most efficient, allowing the observation of the optomagnetic effect as was shown in Fig. 3. By finetuning of the external field one may balance the magnetic domains more precisely and thus suppress the observation of the heat-driven effect completely. At a laser fluence of $2 \mathrm{~mJ} / \mathrm{cm}^{2}$, the ratio $\Delta M_{\text {opto }} / \Delta M_{\text {heat }}$ was estimated to be about 0.21 [22].

The observed optomagnetic effect can be described under the assumption that the effect of the laser pulse on the magnetic system is equivalent to the action of an equally long (100 fs) magnetic field pulse $\mathbf{H}_{F}$ directed along the wave vector of light $[14,15,18]$. This effective field pulse along the $z$ axis triggers a precession of the magnetization, but now the $z$ components of $\mathbf{M}_{1}$ and $\mathbf{M}_{2}$ oscillate in phase, in contrast to the heat-driven effect where the $z$ components of the magnetization in the two opposite domains oscillate out of phase (see inset Fig. 4). As a result, the optomagnetic effect can be detected.

This optomagnetic effect is suggested to be due to the inverse Faraday effect [23,24], which can phenomenologically be described as an axial magnetic field

$$
\mathbf{H}(0)=(\chi / 16 \pi)\left[\mathbf{E}(\omega) \times \mathbf{E}^{*}(\omega)\right],
$$

where $\mathbf{E}(\omega)$ and $\mathbf{E}^{*}(\omega)$ are the electric field of the light wave and its complex conjugate, respectively; $\chi$ is the 
magneto-optical susceptibility. Equation (1) indicates that the axial magnetic field is induced by circularly polarized light along its $\mathbf{k}$ vector. In addition, the sign of this induced field depends on the helicity of light $[14,15,19]$.

$\Delta M_{\text {heat }}$ and $\Delta M_{\text {opto }}$ separate two completely different effects which are coexisting in this metallic system, following laser excitation. While the heat-driven excitation is based on the absorption of light, the inverse Faraday effect is due to the ultrafast interaction of the photon angular momentum with the magnetic system, taking place via a Raman-like coherent optical scattering process [14,18,25]. Thus, this optomagnetic mechanism does not require annihilation of a photon. Instead, the photons stimulate the transfer of the required angular momentum from the lattice to the spins. As the energy loss per scattering event is very small ( $\hbar \omega_{\text {magnon }} \ll$ photon energy), one single photon can effectively interact with more than one spin, explaining the efficiency of this effect for metallic materials. It is observed from Eq. (1) that the strength of the effective magnetic field $\mathbf{H}_{F}$ depends on the laser fluence. Although in our experiments we used a relatively high laser fluence for a metallic magnetic material $\left(2 \mathrm{~mJ} / \mathrm{cm}^{2}\right)$, this is still low compared with the experiments on nonabsorbing dielectrics (e.g., $60 \mathrm{~mJ} / \mathrm{cm}^{2}$ [18]) where a strong optomagnetic effect was observed. Therefore, the usual experimental conditions on metallic magnets, of low laser fluence in addition to a strong heat-driven effect, require a special technique for the detection of ultrafast optomagnetic effects, as was shown here. On the other hand, under the proper conditions, the effect of the angular momentum can be fully exploited [26]. We believe that the observation of the inverse Faraday effect in a metallic magnet together with the previously reported observation of this effect in orthoferrites [14,19] and garnets [15] indicate that this mechanism does not rely on specific material properties but is a general phenomenon (see also [20]). Thus, it must also occur in ferromagnetic materials such as $\mathrm{Ni}$.

In conclusion, by using an all-optical pump-probe setup and turning the GdFeCo alloy into a state with small magnetic domains, we have been able to average out the laser induced heating effects in the Faraday signal. Consequently, we have observed that photoexcitation with right- and left-handed circularly polarized laser pulses results in spin precessions of different phase. This observation clearly demonstrates that in the metallic ferrimagnetic alloy $\mathrm{GdFeCo}$ an ultrafast nonthermal coupling between spins and the angular momentum of photons exists.

The authors acknowledge the technical support of T. Toonen, W. Szweryn, and A. van Etteger and the financial support of The European network DYNAMICS, FOM, NWO, and the Grant in Aid for Scientific Research of the MEXT in Japan No. 16360182.

*Electronic address: d.stanciu@science.ru.nl

[1] J. Stohr and H.C. Siegmann, Magnetism: From Fundamentals to Nanoscale Dynamics (Springer-Verlag, Berlin, 2006).

[2] J.-Y. Bigot, C.R. Acad. Sci. Ser. IV (Paris) 2, 1483 (2001).

[3] N. Del Fatti et al., Phys. Rev. B 61, 16956 (2000).

[4] A. Scholl et al., Phys. Rev. Lett. 79, 5146 (1997).

[5] E. Beaurepaire et al., Phys. Rev. Lett. 76, 4250 (1996).

[6] M. Vomir et al., Phys. Rev. Lett. 94, 237601 (2005).

[7] L. Guidoni, Eric Beaurepaire, and Jean-Yves Bigot, Phys. Rev. Lett. 89, 017401 (2002).

[8] A. Melnikov et al., Phys. Rev. Lett. 91, 227403 (2003).

[9] G. P. Zhang and W. Hubner, Phys. Rev. Lett. 85, 3025 (2000).

[10] B. Koopmans et al., Phys. Rev. Lett. 85, 844 (2000).

[11] G. Ju et al., Phys. Rev. B 57, R700 (1998).

[12] V. V. Kruglyak et al., Phys. Rev. B 71, 233104 (2005).

[13] R. Wilks et al., J. Appl. Phys. 95, 7441 (2004).

[14] A. V. Kimel et al., Nature (London) 435, 655 (2005).

[15] F. Hansteen et al., Phys. Rev. Lett. 95, 047402 (2005).

[16] C. D. Stanciu et al., Phys. Rev. B 73, 220402(R) (2006).

[17] J. Hohlfeld et al., Phys. Rev. B 65, 012413 (2001).

[18] F. Hansteen et al., Phys. Rev. B 73, 014421 (2006).

[19] A. V. Kimel et al., Phys. Rev. B 74, 060403(R) (2006).

[20] R. Hertel, J. Magn. Magn. Mater. 303, L1 (2006).

[21] Te-ho Wu, J. Appl. Phys. 81, 5321 (1997); M. Kisielewski et al., J. Appl. Phys. 93, 6966 (2003).

[22] The magnitude of the heat-driven effect was obtained from the single domain experiment.

[23] L. P. Pitaevskii, Sov. Phys. JETP 12, 1008 (1961).

[24] J.P. van der Ziel, P. S. Pershan, and L. D. Malmstrom, Phys. Rev. Lett. 15, 190 (1965).

[25] P.S. Pershan, J. P. van der Ziel, and L.D. Malmstrom, Phys. Rev. 143, 574 (1966).

[26] Recently, we demonstrated that the magnetization in metallic materials can be reversed in a reproducible manner by a single 40 femtosecond circularly polarized laser pulse, without any applied magnetic field: International patent pending, PCT/NL2006/000264. 